\title{
Comparison between Simulation and Hardware Realization for Different Clock Steering Techniques
}

\author{
Marion Gödel, Tobias D. Schmidt and Johann Furthner \\ Department of Navigation, German Aerospace Center (DLR), Münchener Strasse 20, 82234 \\ Wessling, Germany \\ E-mail: tobias.schmidt@dlr.de
}

\begin{abstract}
Clock steering is an important discipline within the time and frequency community as well as in cognate disciplines such as global navigation satellite systems. In the latter, clock steering is a mandatory tool for realizations of time scales, which first are typically created by algorithms forming a paper clock. Often, this software solution of a time scale is already sufficient. However, sometimes a realization in form of a frequency signal is feasible or even necessary. Future global navigation satellite systems concepts plan to use composite clocks consisting of several different clocks to achieve synchronization of satellites within one orbit with the system time. In this approach, a real clock output in the microwave regime is indispensable. Therefore, a real clock has to be steered against the paper clock to realize the composite clock. Consequently, steering techniques are an essential piece for obtaining a realization. Besides the number of algorithms to choose from, there are several parameters that need to be defined by the user to achieve the best performance for distinct scenarios. In this paper we compare the realization performance and applicability of two different steering techniques, namely linear-quadratic Gaussian control and pole placement, in a simplified hardware setup. The studied scenario consists of a steerable clock consisting of a micro phase stepper which changes the frequency of the output of an oven-controlled quartz oscillator (OCXO) and a rubidium atomic clock (RB). The OCXO in turn is steered to follow the free-running RB as closely as possible. Steering the output of the OCXO introduces a new process in the clock. As a result, the Allan deviation of the steered clock shows a bump which location is dependent on the chosen control interval and steering technique. This has to be considered for the designated operation. We compare simulations of the clock steering with both techniques to real-data results from our laboratory.
\end{abstract}

PACS numbers: 1315, 9440T

keywords: control, steering, pole placement, linear-quadratic Gaussian control, Kalman filter Submitted to: Metrologia 


\section{Introduction}

A stable system time is one essential piece of global navigation satellite systems (GNSSs). Since position determination is based on signal travel times, any error in time will directly propagate into the position estimation. Therefore, an accurate, stable, and robust time scale has to be established. Once determined, all clocks within the system need to be synchronized to this system time. In most existing systems, the ground monitor network derives the correction values for each satellite clock relative to the system time. In the next step, these offsets are uploaded to the constellation and broadcast to users through the navigation message. Any improvement in the system time scale's stability or the satellite clock's stability will enhance the accuracy of the position obtained by the user. One approach to improve the stability of the timing signal is applying a clock ensembling technique to a set of clocks.

Our work focuses on using a Kalman filter based approach to form an ensemble time from several clocks [1]. The filter estimates the individual clock states from differential measurements. The difference between these estimates and the true clock states is weighted using the error covariance matrix from the filter providing the Implicit Ensemble Mean (IEM). The IEM is a paper time scale that outperforms the individual clock contributions in terms of stability. This method was first described by Brown [2] and later adapted by Greenhall [3] to follow the best clock in the ensemble over all averaging times. A review on clock ensembling techniques based on the Kalman filter can be found in [4].

Nevertheless, both approaches use the actual clock states to derive the ensemble mean. Since the actual clock states are only available in simulations, the ensemble mean cannot be accessed in a real scenario. In a recent publication, the authors have demonstrated that the ensemble time can be realized by using a second Kalman filter and a steerable clock that contains a Micro Phase Stepper (MPS) which adapts the frequency output of an oven-controlled quartz oscillator (OCXO)[5]. The estimate of one ensemble clock is combined with the measurement of the OCXO relative to this clock. The combination eliminates the ensemble clock which is present in both quantities and thus yields the difference between the OCXO and the IEM plus distortions. The second Kalman filter is used to estimate the difference between the OCXO and ensemble mean from the noisy input and derives a control value to drive their difference to zero. As a result, the MPS output (based on the OCXO) follows the IEM and serves therefore as a realization of the IEM. The control value, which is applied by the MPS, is obtained using a steering technique, in our previous work the linear-quadratic Gaussian (LQG) control [5].

One important step in the process of realization a time scale generated by an ensembling algorithm is to lock one physical oscillator to a second one. In the case describe above, an OCXO is steered to a software made oscillator, namely the IEM. In this study, we focus on this essential part when realizing a time scale. In order to decouple the impact of the steering performance and the numerical generation of the IEM, we decided to analyze the steering process itself separately. Thus, in this study, we focus on the steering performance of one physical oscillator to another one.

Several steering techniques for frequency standards have been published in the last decades [6]. Farina et al. describe three common techniques: the signal-based open-loop, the GPS bang-bang method, and the linear-quadratic Gaussian control [7]. The signal-based open loop approach is typically used to compensate for the frequency offset, as for example in [8]. The GPS bang-bang algorithm described in [9] was designed to steer GPS time to UTC(USNO). The application of the LQG control technique to steer hydrogen masers to coordinated universal time (UTC) was outlined by Koppang and Leland in [10]. Furthermore, in a recent paper by Koppang, the pole placement (PP) technique is adapted for steering clocks [11]. In addition, several algorithms have been published for the steering of local UTC realizations $[12,13,14,15]$. Recently, we presented a simulative comparison between $\mathrm{PP}$ and LQG for steering an OCXO against a rubidium atomic clock (RB) with focus on the steering performance for different steering parameters, as we want to use PP for future realization of a composite clock based on a mixed clock ensemble [16]. This investigation is the base for the here presented study. In this work, we compare the overall performance of both control techniques in a hardware setup to simulations. The comparison does not show evident differences between the two techniques.

The linear-quadratic Gaussian control approach requires a Kalman filter that estimates the quantity that is supposed to be driven to a certain state. The control value is then obtained by multiplying 
the estimate to the linear-quadratic regulator (LQR), a quantity obtained from the control system. The pole placement technique does not require a Kalman filter but is applied in the same way. The techniques were chosen because they have a similar setup and consequently are easily interchangeable. Thus, the same hardware setup can be used to test both steering techniques needing only minor changes in the software.

Even though for both control techniques simulations with varying parameter sets can be found in literature, there is barely real data published so far. Koppang et al. have shown results of LQG and PP using real clock data with the intention to optimize a system for long-term stability [17]. However, we present the impact of the control interval on the steering performance with real measurement data, as well as compare those results with the expected performance obtained by numerical simulations, which will influence the decision between both steering techniques for future applications.

\section{State of the Art}

First, we will describe the two steering techniques that we haven chosen to analyze, linear-quadratic Gaussian control and pole placement. Both methods require a model for the clock that is supposed to be controlled.

\subsection{Clock Modeling}

The clocks are modeled by the well-established discrete two-state clock model $[18,19]$

$$
x\left(t_{k+1}\right)=\phi(\tau) x\left(t_{k}\right)+w\left(t_{k}\right)
$$

with transition matrix

$$
\phi(\tau)=\left(\begin{array}{ll}
1 & \tau \\
0 & 1
\end{array}\right)
$$

and process noise $w\left(t_{k}\right) \sim \mathcal{N}(0, Q(\tau))$ where $\tau=$ $t_{k+1}-t_{k}$. The two states represent the phase deviation and part of the frequency deviation. The process noise incorporates two different noise types: white frequency noise and random walk frequency noise. The covariance of the process noise

$$
Q(\tau)=\left(\begin{array}{cc}
q_{1} \tau+\frac{q_{2} \tau^{3}}{3} & \frac{q_{2} \tau^{2}}{2} \\
\frac{q_{2} \tau^{2}}{2} & q_{2} \tau
\end{array}\right)
$$

is based on the diffusion coefficients $\sigma_{1}, \sigma_{2}\left(q_{i}=\sigma_{i}^{2}\right.$, i $=1,2)$ which drive the fundamental noises. This model can be used to describe the behavior of a single clock as well as the difference between two clocks $x_{1}$ and $x_{2}$ :

$$
\begin{aligned}
x_{3}\left(t_{k}\right) & =x_{2}\left(t_{k}\right)-x_{1}\left(t_{k}\right) \\
x_{3}\left(t_{k+1}\right) & =\phi(\tau) x_{3}\left(t_{k}\right)+w_{3}\left(t_{k}\right)
\end{aligned}
$$

with $w_{3}\left(t_{k}\right) \sim \mathcal{N}\left(0, Q_{1}(\tau)+Q_{2}(\tau)\right)$, where $Q_{1}(\tau)$ and $Q_{2}(\tau)$ are the process noise covariance matrices for $x_{1}$ and $x_{2}$ respectively. A small process noise covariance matrix indicates a superior clock performance. In the extreme case with no process noise present, we find the theoretical ideal clock which consists purely of a transition from the last state without any perturbation.

The measurement of the clock is modelled by

$$
z\left(t_{k}\right)=H x\left(t_{k}\right)+v\left(t_{k}\right)
$$

where the measurement matrix $H$ describes the structure of the measurement and $v\left(t_{k}\right) \sim \mathcal{N}(0, R)$ is the measurement noise with covariance $R$.

The process model in eq. 1 only describes the behavior of a free-running frequency standard. If control values are applied on the frequency standard, the model has to be extended by a control term $b(\tau) u\left(t_{k}\right)$ to

$$
x\left(t_{k+1}\right)=\phi(\tau) x\left(t_{k}\right)+b(\tau) u\left(t_{k}\right)+w\left(t_{k}\right)
$$

where $u\left(t_{k}\right)$ is the control value and $b(\tau)$ propagates the control value [10]. This change in the process model will only affect the prediction of the state in the Kalman filter formulas where the term $b(\tau) u\left(t_{k}\right)$ needs to be added as well. We will now focus on different methods to find the optimal control value $u\left(t_{k}\right)$ in every time step.

\subsection{Linear-Quadratic Gaussian Control}

For linear-quadratic Gaussian control the feedback gain is chosen such that the cost function

$$
J=\sum_{i=1}^{\infty} x_{i}^{T} W_{Q} x_{i}+u_{i}^{T} W_{R} u_{i}
$$

is minimized, where the first part in the sum penalizes the transient state transition and the second term penalizes the control effort. So depending on the user's choice of $W_{Q}$ and $W_{R}$ the system is either steered gentle or rapid. Once the steering matrices have been identified, the linear-quadratic regulator $\hat{K}_{0}$ is derived by solving the steady-state Ricatti equation

$$
\begin{aligned}
\hat{K}_{0}= & \phi(\tau)^{T} \hat{K}_{0} \phi+W_{Q}- \\
& \phi(\tau)^{T} \hat{K}_{0} b\left(b^{T} \hat{K}_{0} b+W_{R}\right)^{-1} b^{T} \hat{K}_{0} \phi .
\end{aligned}
$$

Solving this equation requires a controllable system. In the next step, one obtains the gain $\hat{G}_{0}=\left(b^{T} \hat{K}_{0} b+\right.$ $\left.W_{R}\right)^{-1} b^{T} \hat{K}_{0} \phi(\tau)$ which is used to derive the control value from the estimate

$$
u\left(t_{k}\right)=-\hat{G}_{0} \hat{x}\left(t_{k}\right) .
$$

The linear-quadratic Gaussian regulator can be obtained from the system parameters $\Phi(\tau), b(\tau)$ and the designer matrices $W_{Q}$ and $W_{R}$. 


\subsection{Pole Placement}

The pole placement technique is designed for a closed loop system. One determines the poles of the system and moves them to the desired pole locations. As shown in $[11,16]$, the poles of a system can be obtained by looking at the eigenvalues of matrix $A$ which defines the transition between two states of the system

$$
\left(\begin{array}{l}
x(t+\tau) \\
y(t+\tau)
\end{array}\right)=A\left(\begin{array}{l}
x(t) \\
y(t)
\end{array}\right) .
$$

Using the extended process model eq. 7, we obtain

$$
\begin{aligned}
\left(\begin{array}{l}
x(t+\tau) \\
y(t+\tau)
\end{array}\right) & =\Phi(\tau)\left(\begin{array}{l}
x(t) \\
y(t)
\end{array}\right)+B u(t) \\
& =\left(\begin{array}{cc}
1-\tau g_{x} & \tau\left(1-g_{y}\right) \\
-g_{x} & 1-g_{y}
\end{array}\right)\left(\begin{array}{l}
x(t) \\
y(t)
\end{array}\right)
\end{aligned}
$$

We find the eigenvalues of $A$ by solving the eigenvalue equation and find the solutions

$\lambda=\frac{\left.-\left(\tau g_{x}+g_{y}-2\right) \pm \sqrt{\left(\tau g_{x}+g_{y}-2\right)^{2}-4\left(1-g_{y}\right)}\right)}{2}$.

Critical damping typically brings the system back to equilibrium conditions in least amount of time and should hence show the best steering performance for our case study. Thus, the aspired poles need to be real and equal. In addition, the absolute values of the poles need to be less than or equal to one to ensure stability $(|\lambda| \leq 1)$. As a result, we obtain the poles

$$
\lambda=\sqrt{1-g_{y}}=1-\sqrt{\tau g_{x}} .
$$

Nevertheless, it should be emphasized that critical damping might not be the best choice for all steering scenarios as sometimes other requirements, such as frequency smoothness or harder control actions are desirable. For a comprehensive control theoretical study of this aspect the reader is referred to a lately published work by Matsakis [20].

\section{Methods}

This study is aimed to provide insights on the steering techniques for the realization of a paper time scale. Nevertheless, to analyze the effects we have decided to reduce the number of influences on the results. Therefore, we are focusing on the straightforward setup of driving one clock to another without applying clock ensembling techniques.

We have empirically found one parameter set for each steering method that yields good results in our scenario for $W_{Q}$ and $W_{R}$ using LQG control and $\lambda$ for pole placement. As we are analyzing the impact of the control interval, we will hold all other parameters

\begin{tabular}{l|l} 
Parameter & Assigned value \\
\hline q-Parameters OCXO & $q_{1}=1.0 \cdot 10^{-24}$, \\
& $q_{2}=5.0 \cdot 10^{-26}$ \\
q-Parameters RB & $q_{1}=1.2 \cdot 10^{-23}$, \\
& $q_{2}=8.0 \cdot 10^{-29}$ \\
Measurement noise & $R=10^{-24}$ \\
Time discretization & \\
of the Kalman filter & $\tau=1$ second \\
LQG control & $W_{Q}=\left(\begin{array}{cc}10^{-4} & 0 \\
0 & 10^{-9}\end{array}\right)$, \\
& $W_{R}=1$ \\
Pole placement & $\lambda=0.85$
\end{tabular}

Table 1: List of parameters used for the simulations and real data runs.

steady. Find a comprehensive list of the parameters in table 1. This table shows the clock parameters that are used for the control loop. For each control interval we have performed four simulations and real data runs of 13 hours to obtain reliable clock stabilities until $10^{4}$ seconds.

The performance of the algorithms is mainly analyzed by the overlapping Allan deviation (OADEV) of the clock performance [21].

\subsection{Sequence of the Steering Process}

Independent of the chosen control interval, the Kalman filter that estimates the difference between the OCXO and the RB based on the measurement, is run every second. The size of the control gains for both methods needs to be adapted to the fact that the control interval is not identical to $\tau$, the time difference between two filter iterations. Therefore, we choose to calculate the control gain for LQG control

$$
\hat{G}_{0}=f\left(\Phi\left(\tau_{c}\right), b\left(\tau_{c}\right), W_{Q}, W_{R}\right)
$$

as well as for PP

$$
g_{x}=\frac{1}{\tau_{c}}(1-\lambda)^{2}, g_{y}=1-\lambda^{2}
$$

dependent on the control interval $\tau_{c}$. This affects the steered clock the same way as if the filter was run in the same frequency as the control values are applied. In iterations where no steering is applied, the last control value is propagated by the transition matrix. Since

$$
\Phi(\tau=1)^{\tau_{c}}=\Phi\left(\tau_{c}\right)
$$

this method leads to the same propagation of the control value as running the filter only on the control interval. The advantage of our methods is that all measurements available are used to predict the difference between the two clocks. 


\subsection{Hardware Setup}

The clocks involved in this investigation are a rubidium atomic clock (SRO-100, Spectratime) and a steerable clock which consists of an oven-controlled quartz oscillator with additional micro phase stepper (KL3382, K+K Messtechnik GmbH). The entire hardware setup carries four identical rubidium atomic clocks and two steerable clocks. However, for the sake of simplicity, we are only using one RB and one steerable clock to proof the hardware applicability and the performance of the steering techniques. Nevertheless, the entire setup allows for IEM realization of composite clocks consisting of all e.g. four RB as in [5].

The phase difference between the two $10 \mathrm{MHz}$ signals of both clocks is measured every second with a time and frequency monitor (KL-3360, Lange electronics). The measurement data is subsequently send to a server (RS-232 connection), on which the Kalman filter for clock steering is performed. As already mentioned in the previous section, the Kalman filter is processed every second while the control value for the steering is calculated and applied to the micro phase stepper after the free selectable control interval time is reached. The steering value is send to the OCXO by an RS-232 connection between the steerable clock and the server.

\subsection{Simulation}

In the simulations, we try to model the behavior of the true clocks in terms of an overlapping Allan deviation as closely as possible. Therefore, we have measured the performance of the OCXO built-in the MPS against an active hydrogen maser (AHM) (CH1$75 \mathrm{~A}$, Kvarz). Please note, that these measurements were taken before the real data runs were started as the hardware does not allow us to monitor the free-running OCXO within the MPS and the steered output signal simultaneously at the moment. The performance of the AHM supersedes the performance of the OCXO at every averaging time by a factor of at least $10^{2}$ (@1s). Therefore, we assume that the OCXO performance will drive the measurement.

The performance of the OCXO is modeled using the two-state model extended by four Markov processes as suggested by Davis et al. [22]. Figure 1 shows the measurement as well as the respective simulation runs. As the simulation is based on statistical appearing noises, we show 20 runs of the simulation to demonstrate the reliability of the simulation. The deviation of the simulation from the measurement at long averaging times is determined by random walk frequency noise. Especially for short averaging times ( $\tau<5$ seconds), not all process noise of the OCXO is

\begin{tabular}{l|l} 
Parameter & Assigned value \\
\hline q-Parameters & $q_{1}=\left(5 \cdot 10^{-12}\right)^{2}$, \\
$q_{2}=5 \cdot 10^{-30}$ \\
$\left.\begin{array}{l}1.89 / 20 \\
1.89 / 200 \\
1.89 /\left(2 \cdot 10^{3}\right) \\
1.89 /\left(2 \cdot 10^{4}\right)\end{array}\right)$ \\
$\begin{array}{l}\text { Positions of the peaks } \\
\text { of the Markov pro- } \\
\text { cesses, } R_{j}\end{array}$ & $\left(\begin{array}{l}4 \cdot 10^{-24} \\
9 \cdot 10^{-24} \\
2 \cdot 10^{-23} \\
5 \cdot 10^{-23}\end{array}\right)$ \\
$\begin{array}{l}\text { Size of the Markov } \\
\text { processes, } U_{j}\end{array}$
\end{tabular}

Table 2: List of parameters for the OCXO model.

reflected in the model. Nevertheless, since we focus on control intervals $\tau_{0} \geq 10$ seconds, it does not impact the stability of the steered clock. The detailed parameter set for the OCXO model can be found in Table 2.

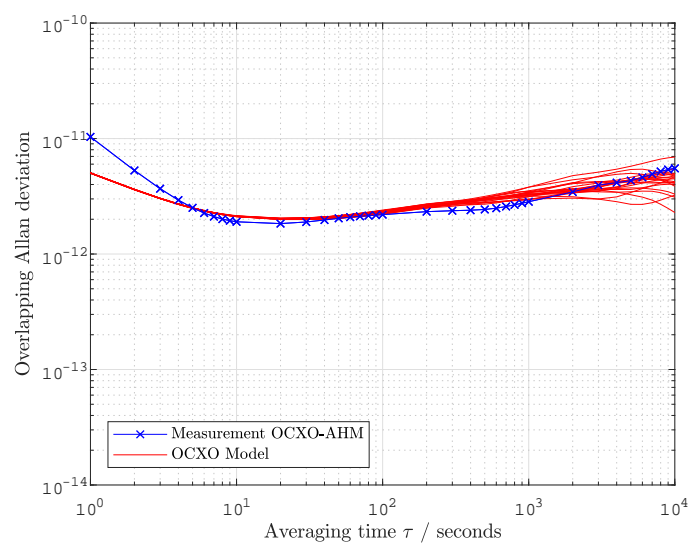

Figure 1: Measurement of the performance of the free-running OCXO against an active hydrogen maser (AHM) together with the simulation of the fitted model (20 simulations).

We are trying to emulate the real situation as closely as possible in the simulation. For the realtime routines, we do not have any information about the free-run OCXO behavior during processing. As a result, there might be a discrepancy between the freerun behavior of the OCXO and the chosen model. Even though we could easily solve this issue in simulations, we decided to use the same parameter set for real data as for simulation. Consequently, we will have a discrepancy between the simulated OCXO and the model used in the Kalman filter. This resembles the situation with real data.

The same holds for the rubidium atomic clock. The advantage is that we can monitor the RB during the real data runs. In figure 2 the measurement of the rubidium atomic clock again against the AHM is 


\begin{tabular}{l|l} 
Parameter & Assigned value \\
\hline q-Parameters & $q_{1}=\left(3.8 \cdot 10^{-12}\right)^{2}$, \\
& $q_{2}=3.5 \cdot 10^{-28}$
\end{tabular}

Table 3: Parameter for the rubidium atomic clock model.

depicted together with 20 simulations of the chosen two-state-model. Table 3 lists the parameters of the model for the RB performance.

As baseline for the performance of the rubidium atomic clock that serves as a reference we have used the measurement of the $\mathrm{RB}$ relative to an active hydrogen maser throughout the period when the real runs where performed.

For the measurement noise $R$, we measured a clock against itself with the time and frequency monitor. This data was then fit to a model of the device's measurement noise.

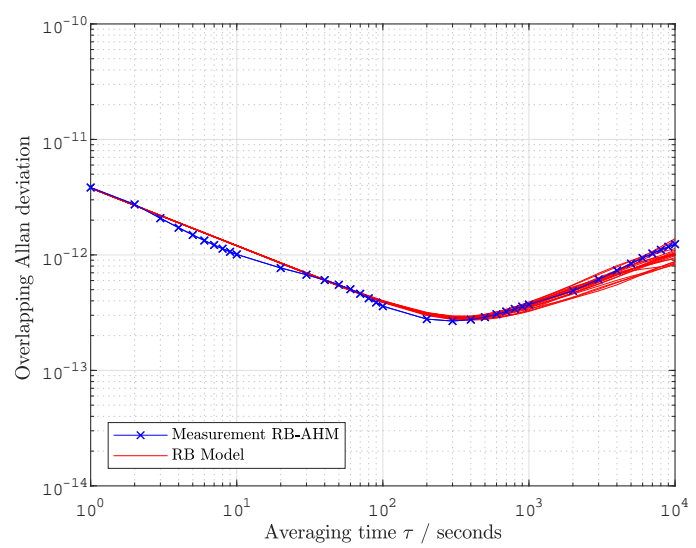

Figure 2: Measurement of the performance of the rubidium atomic clock against an active hydrogen maser (AHM) together with the simulation of the fitted model (20 simulations).

\section{Results and Discussion}

For both steering techniques we have carried out four real data runs for a time period of 13 hours and four simulations for the following control intervals: $1,2,10$, 20 and 100 seconds. For the sake of convenience we first discuss the results for linear-quadratic Gaussian control, then the results for pole placement.

For each data set, three curves are plotted in terms of an OADEV analysis: First, the monitoring of the free-running $\mathrm{RB}$ relative to an active hydrogen maser. Second, the monitoring of the steered OCXO relative to the AHM. Third, the difference between the rubidium atomic clock and the quartz clock. Whereas the first two graphs are assumed to show the individual performance of the RB and the OCXO, respectively, the third graph is based on the only measurement that is directly available from the setup. This measurement is fed into the Kalman filter every second. In case of the simulations, the same quantities will be shown but from the simulated clocks. In addition, the performance of the OCXO is plotted for a time frame in which it was not manually adjusted but in free-run mode.

The direct investigation of the phase differences is complicated to analyze since even small deviations can lead to major differences in stability. Thus a more robust and comprehensive method is required to evaluate the steering process. We will base our analysis mainly on the stability analysis in terms of an OADEV of the differential phase measurement between the two frequency standards. As soon as both clocks are syntonized, the phase difference between them does not undergo changes anymore. As a consequence, the stability of the phase difference is dominated by white frequency modulation noise, which results in a constant slope of -1 in a log-log scale OADEV plot. An appearance of this feature over a wide range over averaging times demonstrates a successful steering.

A certain behavior of the steered clock is expected: While the averaging time is smaller than the control interval $\tau<\tau_{c}$, the OCXO should not be impacted by the steering process and therefore be identical to its free-run performance. Once the averaging times are greater than the control interval, the stability of the steered OCXO signal is deviating from its freerun. Around this averaging time, typically a bump in the stability can be observed before the stability of the steerable clock converges to the reference, the rubidium atomic clock. However, it takes some averaging time until the stabilities of both signals are aligned in an OADEV analysis. The reason is the inferior stability of the steered clock compared to the reference at $\tau=\tau_{c}$. So, the steered clock thereafter needs some time to reach the reference clock performance.

\subsection{Linear-Quadratic Gaussian Control}

At first we will discuss the results obtained with the linear-quadratic Gaussian control and thereafter the pole placement technique. In figure 3 the results of one real data run with linear-quadratic Gaussian control is depicted for a control interval of 20 seconds. The performance of the steered OCXO aligns to the performance of the free-running rubidium atomic clock after an averaging time of roughly $10^{3}$ seconds. That means, the same noise types are present in both clocks. Furthermore, the slope of the stability of the differential phase measurement between both clocks exhibit a slope of -1 for averaging times $\tau>10^{2}$. Once the steering of the quartz clock is successful, the performance of the RB and the OCXO are identical 


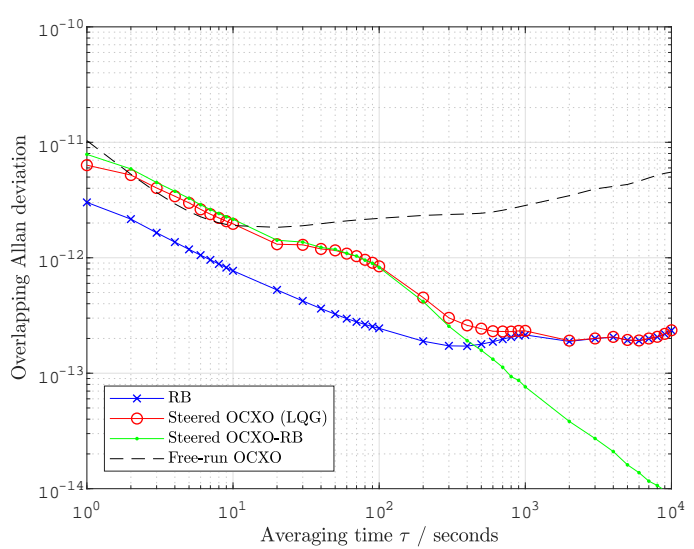

Figure 3: Evaluation of the LQG control technique. A single real data run was performed using a control interval of 20 seconds. The OCXO (red circles) is steered to the RB (blue crosses). The stability of the measurement between OCXO and RB (green solid line) exhibits a slope of -1 once the clocks are aligned. For comparison, the free-run stability of the OCXO is shown (black dashed line).

and the difference between them shows a slope of -1 in the OADEV analysis (white noise). In addition, the performance of the free-running OCXO is shown. The stability of the steered OCXO is identical to its free-run performance for $\tau \in\left[10^{0}, 10^{1}\right]$. This behavior is expected as the control interval was set to $20 \mathrm{~s}$. Thus, the short-term performance of the OCXO should not be impacted by the steering. Consequently it is following the performance of the free-running OCXO signal.

In order to demonstrate the reliability and the statistical significance of the demonstrated steering performance figure 4 displays the evaluation of four different hardware runs in terms of overlapping Allan deviation. All runs were performed with identical steering values including the control interval of $20 \mathrm{~s}$. They show similar steering performances, what demonstrates that the method is sufficient to steer the OCXO to the rubidium atomic clocks' signal.

In the next step, we want to compare the hardware runs to simulations. Figure 5 demonstrates the expected steering performance for the LQG steering technique using a control interval of $20 \mathrm{~s}$ obtained from numerical simulations. Although the rubidium atomic clock performances in the four simulation runs are significantly more aligned compared to the real measurements, the steering performance of the OCXO signal is equivalent. Not only the averaging time from which on the phase difference analysis in terms of OADEV shows pronounced white frequency noise (slope of -1 in the plot) but also the averaging time at which the stability evaluation of the steerable clock

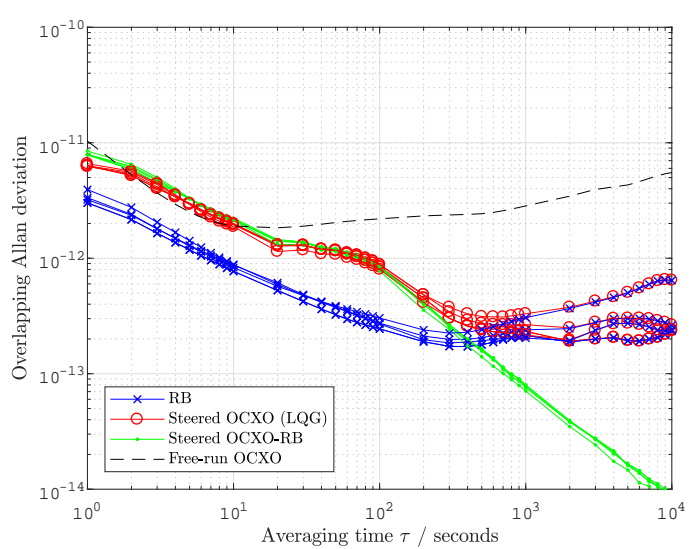

Figure 4: Evaluation of the LQG control technique. Four real data run were performed using a control interval of 20 seconds. The OCXO (red circles) is steered to the RB (blue crosses). The stability of the measurement between OCXO and RB (green solid line) exhibits a slope of -1 once the clocks are aligned. For comparison, the free-run stability of the OCXO is shown (black dashed line).

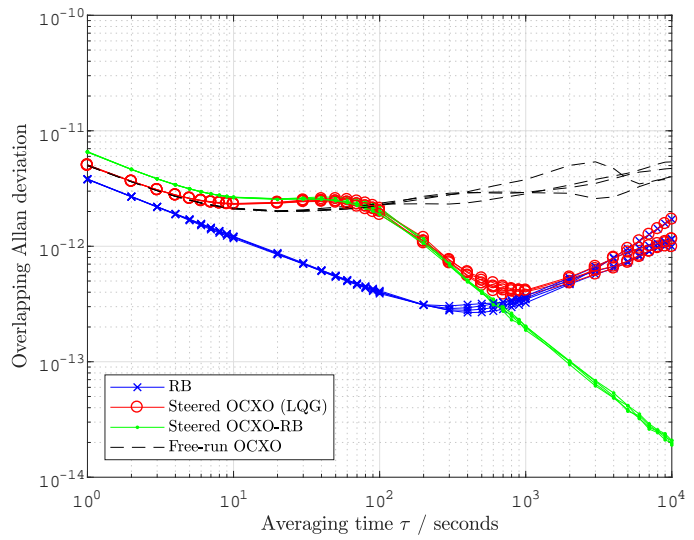

Figure 5: Evaluation of the LQG control technique. Four simulations were performed using a control interval of 20 seconds. The OCXO (red circles) is steered to the RB (blue crosses). The stability of the measurement between OCXO and RB (green solid line) exhibits a slope of -1 once the clocks are aligned. For comparison, the free-run stability of the OCXO is shown (black dashed line).

is aligned to the rubidium atomic clock are in high agreement for simulations and real data measurements. Moreover, the expected bump in the performance of the steered OCXO signal, which originates from the steering process itself, is at the same position although the height is less pronounced in the real data. This might be a result of slightly different OCXO performance for the real runs compared to the simulations. As described in section 3.2, we are currently not able to measure the free-run performance 


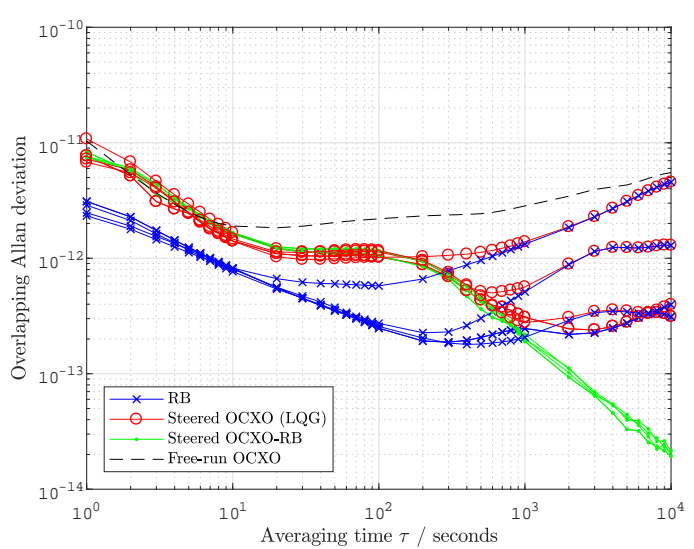

Figure 6: Evaluation of the LQG control technique. Four real data runs were performed using a control interval of 100 seconds. The OCXO (red circles) is steered to the RB (blue crosses). The stability of the measurement between OCXO and RB (green solid line) exhibits a slope of -1 once the clocks are aligned. For comparison, the free-run stability of the OCXO is shown (black dashed line).

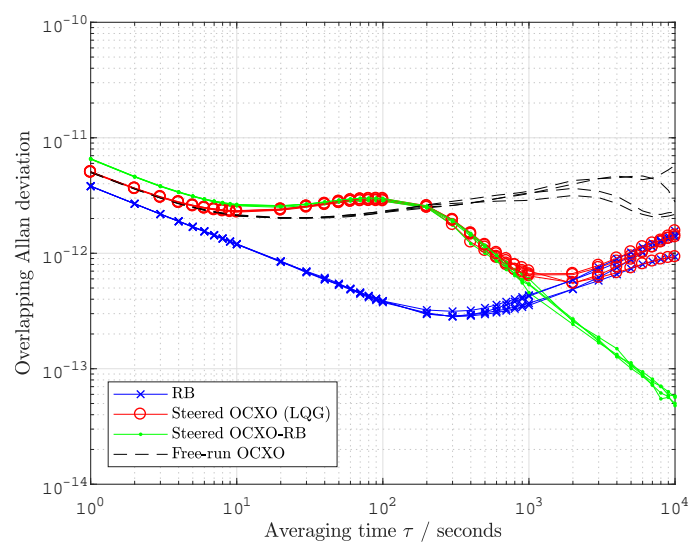

Figure 7: Evaluation of the LQG control technique. Four simulations were performed using a control interval of 100 seconds. The OCXO (red circles) is steered to the RB (blue crosses). The stability of the measurement between OCXO and RB (green solid line) exhibits a slope of -1 once the clocks are aligned. For comparison, the free-run stability of the OCXO is shown (black dashed line).

of the OCXO while a steering is performed on the MPS. Consequently, we can only speculate about the origin of the deviations between simulation and real measurements. The performance of all clocks within the setup is within specification. Nevertheless, the agreement between simulations and real measurements is significant so that we are confident that the steering process works as expected.

With a focus on the differences introduced by different control intervals, we will now take a look

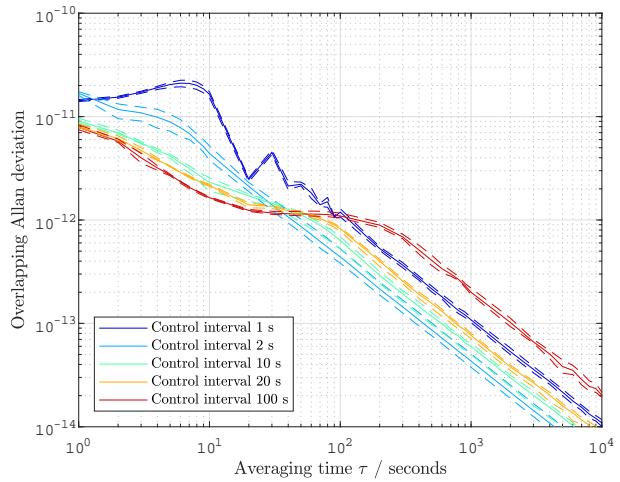

Figure 8: Evaluation of the clock stability for different control intervals using LQG control. Four real data runs were performed for each control interval. For each control interval, the mean (full line) and standard deviation (dashed line) of the measurement between rubidium atomic clock and OCXO over all runs are shown.

at the steering performance for a control interval of $100 \mathrm{~s}$. Again, we performed four individual runs of simulations as well as hardware measurements. Figure 6 is showing the results of the hardware runs. The results are similar to the scenario with the control interval of $20 \mathrm{~s}$. However, the steering takes place for higher averaging times and consequently the syntonization is observed later around an averaging time of $300 \mathrm{~s}$. Even though the overall performance of the RB was not as stable as in the previous runs, the steering worked sufficient. The steered OCXO performance aligns with the stability of the rubidium atomic clock for averaging times over approximately $10^{3} \mathrm{~s}$.

To ensure that proper steering performance was achieved, these results are compared to numerical simulations. Figure 7 shows the behavior of the free-running $\mathrm{RB}$ as well the steered OCXO in four individual simulation runs. Analogue to the control interval of $20 \mathrm{~s}$ the investigations of the performance shows a good agreement between simulation and real data measurement for a control interval of $100 \mathrm{~s}$. Only the rubidium atomic clock exhibits slightly different stability behavior in the hardware measurements. One possible explanation is that during the time period of the real measurements the environmental conditions slightly changed in the laboratory.

In order to further investigate the steering performance as a function of the control interval, we take a closer look at the real data runs with different control intervals. We calculated the mean and the standard deviation of the OADEV behavior of the phase difference between the rubidium atomic clock and the steered OCXO for five different control 


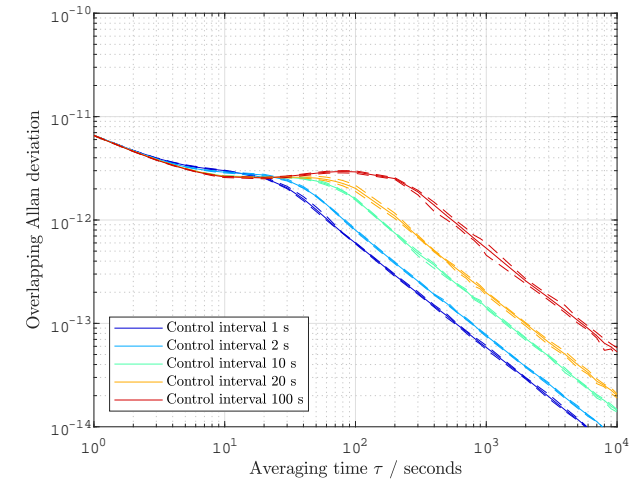

Figure 9: Evaluation of the clock stability for different control intervals using LQG control. Four simulations were performed for each control interval. For each control interval, the mean (full line) and standard deviation (dashed line) of the measurement between rubidium atomic clock and OCXO over all runs are shown.

intervals between 1 and 100 seconds. Figure 8 shows the results. It can easily be seen that the magnitude and location of the steering introduced bump is dependent on the control interval. In particular, the bump is most distinct if we apply the control values every second to achieve rapid steering. For the largest control interval of 100 seconds, we can barely identify a bump, due to the smooth steering process. Nevertheless, the averaging time from which on we identify syntonization between both clocks (slope of -1 in the OADEV plot) occurs later with increasing control interval. That means it takes longer to drive the OCXO to the rubidium atomic clock. Consequently, the control interval needs to be chosen in accordance with the other control parameters to ensure sufficient steering for the distinct scenario.

In addition, we can now compare these results with the expected dependence on the control interval from simulations. Figure 9 displays the simulated behaviors of the phase differences between the reference and the steered OCXO. The simulations for the control intervals between 10 and $100 \mathrm{~s}$ are in good agreement with the real data measurements. However, the scenarios for the control intervals of 1 and $2 \mathrm{~s}$ show significant deviations. Here, no pronounced steering bump is visible in the simulations, while the largest bump appears for a control interval of $1 \mathrm{~s}$ in the real data measurements. We suggest that this behavior is caused by not sufficient communication between the measurement devices and the algorithms as the measurements are taken every second but need some additional time until they are proceeded to the algorithm routines. This might lead to problems with the state updates in the Kalman filter, as these

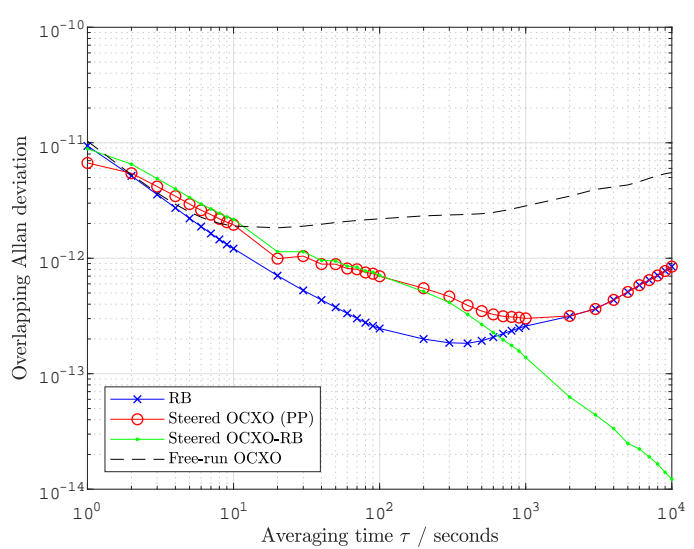

Figure 10: Evaluation of the pole placement technique. A single real data run was performed using a control interval of 20 seconds. The OCXO (red circles) is steered to the RB (blue crosses). The stability of the measurement between OCXO and RB (green solid line) exhibits a slope of -1 once the clocks are aligned. For comparison, the free-run stability of the OCXO is shown (black dashed line).

values are calculated every second, too. These issues can presumably be overcome by establishing faster communications between the devices or increasing the control intervals. Nevertheless, the good agreement between simulations and measurements allows for an optimization of a distinct hardware steering scenario by performing numerical simulations in advance which are much less time consuming.

\subsection{Pole Placement}

Analogous as for LQG control, we will first take a look at the results of the steering process using pole placement and a control interval of $20 \mathrm{~s}$. In figure 10 the stability of the steered OCXO and the free-running rubidium atomic clock, both monitored against an active hydrogen maser, are shown together with the stability of the phase difference measurement between the clocks. Furthermore, the performance of the freerunning OCXO detected before the steering test took place is shown.

Interestingly, the pole placement technique shows similar steering performance than the linear-quadratic Gaussian control for the chosen control parameter. However, two slight differences are observable: First, the syntonization takes place for lager averaging times compared to the LQG run for a control interval of $20 \mathrm{~s}$. Nevertheless, it seems that the converging of the steered OCXO to the reference performance thereafter is faster, so that the alignment of the stabilities of the RB and the OCXO happens for nearly identical averaging times. Second, the bump 


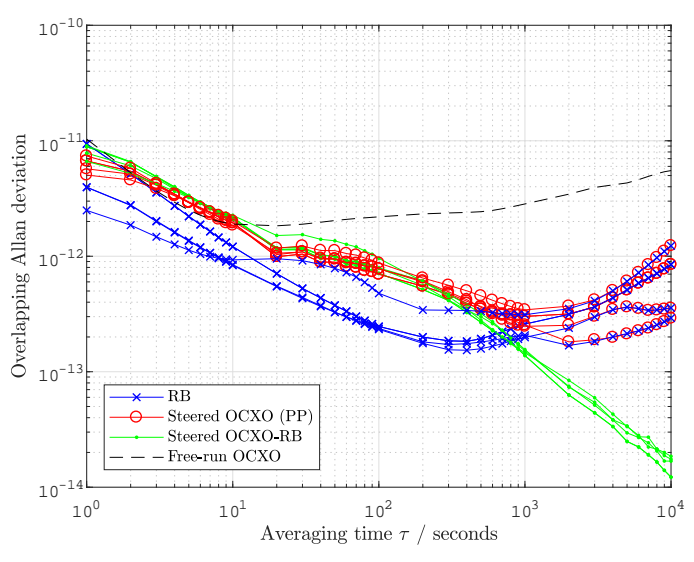

Figure 11: Evaluation of the pole placement technique. Four real data runs were performed using a control interval of 20 seconds. The OCXO (red circles) is steered to the RB (blue crosses). The stability of the measurement between OCXO and RB (green solid line) exhibits a slope of -1 once the clocks are aligned. For comparison, the free-run stability of the OCXO is shown (black dashed line).

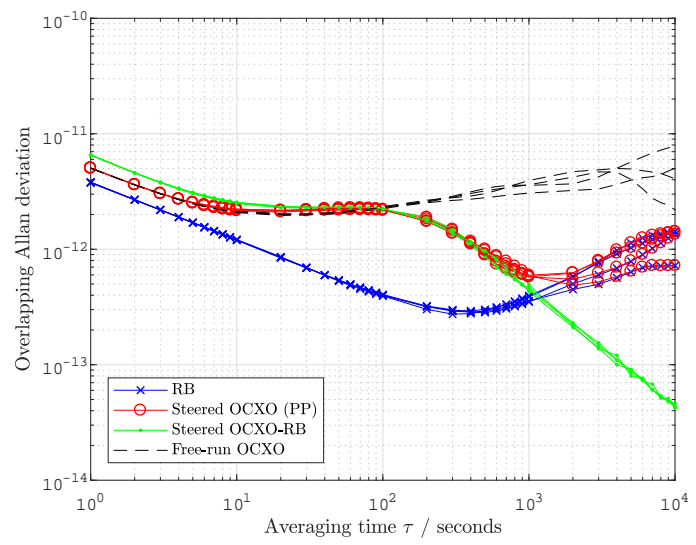

Figure 12: Evaluation of the pole placement technique. Four simulations were performed using a control interval of 20 seconds. The OCXO (red circles) is steered to the RB (blue crosses). The stability of the measurement between OCXO and RB (green solid line) exhibits a slope of -1 once the clocks are aligned. For comparison, the free-run stability of the OCXO is shown (black dashed line).

in the stability behavior of the steered OCXO, which is introduced by the steering process itself, is much less pronounced in the pole placement case. Both differences might originate in the prior chosen other steering parameters, as we use a slightly gentler steering in the pole placement case compared to the linear-quadratic Gaussian control. For more details about the steering performance using different control values for a constant control interval based on simulations it is referred to Ref. [16]. However, the overall steering performance of the pole placement technique is similar to the presented LQG scenario.

To demonstrate sufficient steering despite the diversification of the clock performances, we again show for different runs of real data measurements as well as simulations in figures 11 and 12, respectively. All real data measurement runs show reproducible steering behaviors although the rubidium atomic clock stability exhibits stronger variations than in the previous runs. Unfortunately, the reason of this unstable behavior is not known. It might be related to environmental changes or similar influences. Nevertheless, it is even more remarkable that the steering process was successful in every run although the used clock model did not represent the real rubidium atomic clock behavior in an accurate way.

The simulations of the steering process in figure 12 again are in good agreement with the real data measurements. The deviation of the steered OCXO stability in term of an OADEV analysis from the supposed free-running OCXO behavior takes place earlier than expected from the simulations. This might be caused by a different performance of the free-running OCXO. Please note that this data originates from a time period before the real data runs with steering were performed. However, the other characteristic features of the steering procedure are well represented in the real data measurement runs. The appearance of the syntonization as well as the alignment of the stability performance of the steered OCXO and the RB takes place at similar averaging times in both cases, simulation and real data measurements. Hence, we achieved sufficient accordance between real measurements and simulations.

The same statement holds for the investigation of pole placement as steering technique using a control interval of $100 \mathrm{~s}$. Figures 13 and 14 demonstrate the performance of the steered OCXO and the rubidium atomic clock using real data measurements as well as simulations, respectively. As expected, the syntonization takes place in the real data measurement runs at higher averaging times around $10^{3} \mathrm{~s}$. The alignment to the reference clock stability is achieved near $5 \cdot 10^{3} \mathrm{~s}$ averaging time. Both results are in good agreement with the simulations.

However, the overall steering performance of the pole placement technique for a control interval of $100 \mathrm{~s}$ is not as good as the runs using linearquadratic Gaussian control as syntonization and stability alignment are happening here for considerably lower averaging times. Nevertheless, the steering with pole placement still shows sufficient performance.

Finally, the steering performance of pole placement for different control intervals between 1 and 


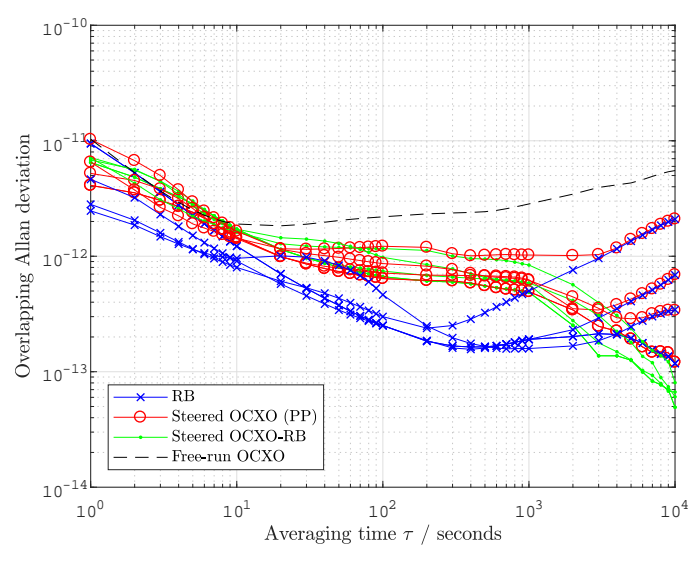

Figure 13: Evaluation of the pole placement technique. Four real data runs were performed using a control interval of 100 seconds. The OCXO (red circles) is steered to the RB (blue crosses). The stability of the measurement between OCXO and RB (green solid line) exhibits a slope of -1 once the clocks are aligned. For comparison, the free-run stability of the OCXO is shown (black dashed line).

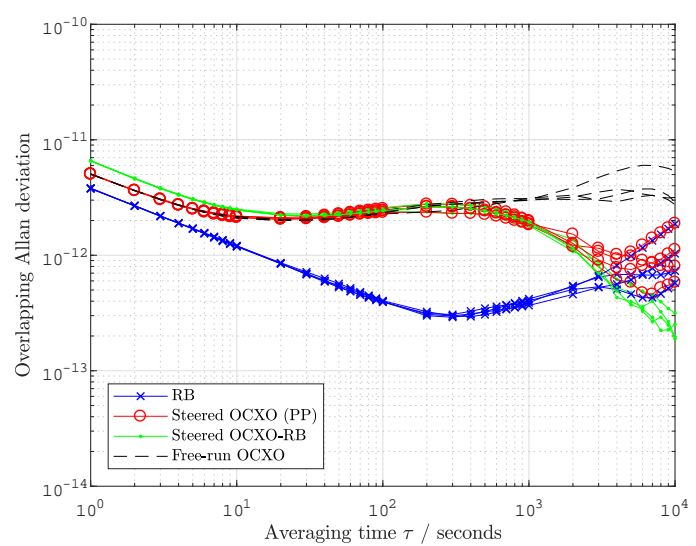

Figure 14: Evaluation of the pole placement technique. Four simulations were performed using a control interval of 100 seconds. The OCXO (red circles) is steered to the RB (blue crosses). The stability of the measurement between OCXO and RB (green solid line) exhibits a slope of -1 once the clocks are aligned. For comparison, the free-run stability of the OCXO is shown (black dashed line).

$100 \mathrm{~s}$ is analyzed. Figures 15 and 16 demonstrate the performance of the phase difference between the steered OCXO and the rubidium atomic clock using real data measurements as well as simulations, respectively. Analogous to the LQG evaluation, the measurements with the control interval of 1 and $2 \mathrm{~s}$ show a pronounced bump in the phase difference analysis using an OADEV characterization. As mentioned before, this feature may show up due to communication delays between the measurement devices and the server running the for the steering responsible Kalman filters. Moreover, the agreement between the simulations and the real data analysis inferior to the LQG runs. One possible explanation is the much higher diversity of the rubidium atomic clock performances during the real measurement runs compared to the LQG runs. This might influence the analysis for the different control intervals in a negative way. Nevertheless, the trend, that the syntonization takes place at higher averaging times for increasing control intervals is clearly visible. Overall, the pole placement technique achieved comparable results to the linear-quadratic Gaussian control scenario and yields sufficient steering.

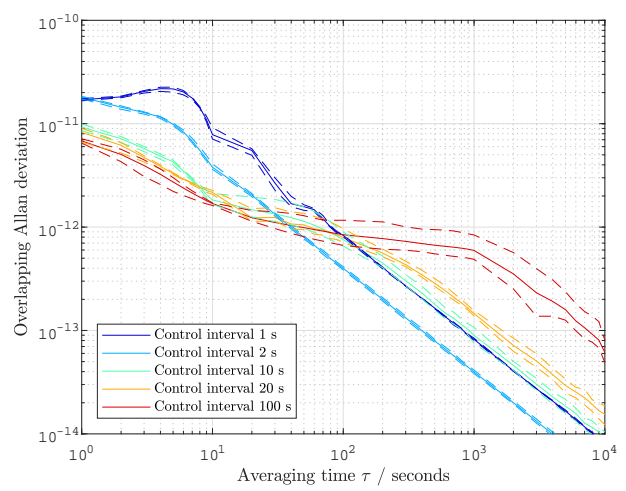

Figure 15: Evaluation of the clock stability for different control intervals using pole placement. Four real data runs were performed for each control interval. For each control interval, the mean (full line) and standard deviation (dashed line) of the measurement between rubidium atomic clock and OCXO over all runs are shown.

\section{Conclusion and Outlook}

In this work, we have presented results of aligning a steerable clock comprised of an OCXO and a micro phase stepper to an RB with two different control techniques. For both techniques, Linear Quadratic Gaussian control and pole placement, the resulting clock stabilities from real data runs were compared to ones retrieved from simulation. There are two main conclusions: First, the steering works sufficient for both techniques even if the free-run performance of the clocks changes. Nevertheless, both techniques require a priori information about the free-run performances of the clocks. The robustness of the algorithms allows an application of the method even if the freerun performance is not fully known. Second, the simulations are in agreement with the real data runs. Since the hardware runs are time consuming, this is beneficial for potential users. They can find the best parameter set for their application using simulations 


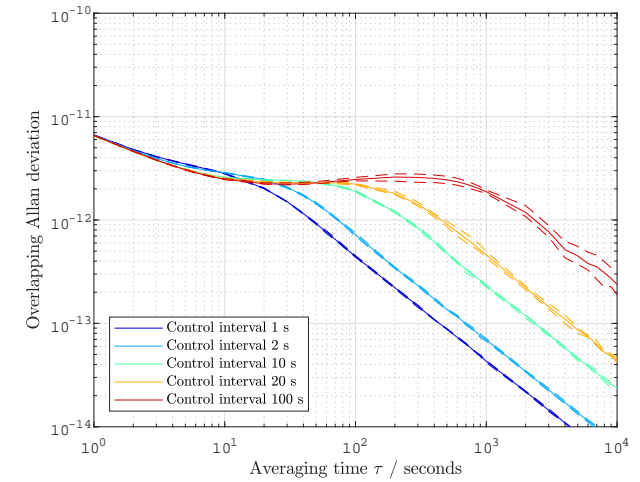

Figure 16: Evaluation of the clock stability for different control intervals using pole placement. Four simulations were performed for each control interval. For each control interval, the mean (full line) and standard deviation (dashed line) of the measurement between rubidium atomic clock and OCXO over all runs are shown.

instead of performing several real data runs. In general, we have shown that the control interval is a dominant parameter of clock steering. The results shown in the paper together with the explanations of the simulation setup supports users to choose the best control interval for their application.

It is noted, that our results do not allow to favor one of the investigated steering methods. Both methods achieve comparable results and thus the selection of one distinct method depends highly on the concrete situation of application.

We have observed that the steering introduces a new process that manifests in the overlapping Allan deviation as bump to the steerable clock's performance. The characteristic of the bump differed between simulation and real data runs. Future investigations will analyze this behavior in more detail as soon as our measurement setup is extended in a way that it will be possible to measure the free-running OCXO and the steered output by the micro phase stepper at the same time.

In addition, there are several issues which need to be handled when realizing a timescale that was generated by a software solution like the Kalman filter as the processing of outliers. At this early stage, we have not addressed these challenges. Nevertheless, these are important points to consider in a real-world system.

\section{Acknowledgement}

Funding by the Helmholtz Association of German Research Centers within the ADVANTAGE project (Contract No. ZT-0007) and DLR project Navigation
4.0 is gratefully acknowledged. The authors would like to thank Dr. Demetrios Matsakis for technical discussions and insights on the steering techniques.

\section{References}

[1] R. E. Kalman, "A new approach to linear filtering and prediction problems," Transactions of the ASME-Journal of Basic Engineering, vol. 82, no. Series D, pp. 35-45, 1960.

[2] K. R. Brown, "The theory of the GPS Composite Clock," in Proceedings of the 4th International Technical Meeting of the Satellite Division of The Institute of Navigation (ION GPS 1991). Institute of Navigation, September 1991, pp. 223242.

[3] C. A. Greenhall, "A Kalman filter clock ensemble algorithm that admits measurement noise," Metrologia, vol. 43, no. 4, pp. S311-S321, 2006.

[4] —, "A review of reduced Kalman filters for clock ensembles," IEEE Transactions on Ultrasonics, Ferroelectrics, and Frequency Control, vol. 59, no. 3, pp. 491-496, 2012.

[5] M. Gödel and J. Furthner, "Robust ensemble time onboard a satellite," in Proceedings of the 48th Annual Precise Time and Time Interval Systems and Applications Meeting, Monterey, California, January 2017, pp. 26-43.

[6] S. Bittanti, M. Farina, L. Galleani, and P. Tavella, "Steering a time scale by the application of the theory of control," in Proceedings of the 19th European Frequency and Time Forum, March 2005, pp. 464-471.

[7] M. Farina, L. Galleani, P. Tavella, and S. Bittanti, "A control theory approach to clock steering techniques," IEEE Transactions on Ultrasonics, Ferroelectrics and Frequency Control, vol. 57, no. 10, pp. 2257-2270, 2010.

[8] P. Tavella and C. Thomas, "Comparative study of time scale algorithms," Metrologia, vol. 28, no. 2, pp. 57-63, 1991.

[9] S. J. Huser and S. T. Hutsell, "1998 GPS time transfer performance," in Proceedings of the 30th Annual Precise Time and Time Interval (PTTI) Systems and Applications Meeting, Reston, VA, Dec 1998, pp. 125-143.

[10] P. Koppang and R. Leland, "Linear quadratic stochastic control of atomic hydrogen masers," IEEE Transactions on Ultrasonics, Ferroelectrics and Frequency Control, vol. 46, no. 3, pp. 517-522, 1999.

[11] P. A. Koppang, "State space control of frequency standards," Metrologia, vol. 53, no. 3, pp. 60-63, 2016 . 
[12] S.-W. Lee, C.-B. Lee, and S. H. Yang, "A new approach for steering UTC(KRIS)," in Conference on Precision Electromagnetic Measurements Digest (CPEM), June 2008, pp. 322-323.

[13] A. Bauch, D. Piester, and E. Staliuniene, "A new realization strategy for the time scale UTC(PTB)," in Proceedings of the 2004 IEEE International Frequency Control Symposium and Exposition, Aug 2004, pp. 518-523.

[14] A. Bauch, S. Weyers, D. Piester, E. Staliuniene, and W. Yang, "Generation of UTC(PTB) as a fountain-clock based time scale," Metrologia, vol. 49, no. 3, pp. 180-188, 2012.

[15] P. A. Koppang and D. N. Matsakis, "New steering strategies for the USNO master clocks," in Proceedings of the 31st Annual Precise Time and Time Interval (PTTI) Meeting, Dana Point, CA, Dec 1999, pp. 277-284.

[16] T. D. Schmidt, M. Gödel, and J. Furthner, "Investigation of pole placement technique for clock steering," in Proceedings of the 49th Annual Precise Time and Time Interval Systems and Applications Meeting, Reston, Virginia, January 2018, pp. 22-29.

[17] P. Koppang, D. Johns, and J. Skinner, "Application of control theory in the formation of a timescale," in Proceedings of the 35th Annual Precise Time and Time Interval (PTTI) Meeting, Sep 2004, pp. 319-324.

[18] C. Zucca and P. Tavella, "The clock model and its relationship with the Allan and related variances," IEEE Transactions on Ultrasonics, Ferroeletrics, and Frequency Control, vol. 52, no. 2, pp. 289-296, 2005.

[19] L. Galleani, L. Sacerdote, P. Tavella, and C. Zucca, "A mathematical model for the atomic clock error," Metrologia, vol. 40, no. 3, pp. 257264, 2003.

[20] D. Matsakis, "The Unification of pole Placement and LQG Steering Techniques," 2019, submitted to: Proceedings of the 50th Annual Precise Time and Time Interval Systems and Applications Meeting.

[21] D. W. Allan, "Time and frequency (time-domain) characterization, estimation, and prediction of precision clocks and oscillators," IEEE Transactions on Ultrasonics, Ferroelectrics and Frequency Control, vol. 34, no. 6, pp. 647-654, 1987.

[22] J. A. Davis, C. A. Greenhall, and P. W. Stacey, "A Kalman filter clock algorithm for use in the presence of flicker frequency modulation noise," Metrologia, vol. 42, no. 1, pp. 1-10, 2005. 